\title{
Revista da Sociedade Brasileira de Medicina Tropical Volume 41(5): página 505
}

A Página 505 do Relato de Caso "Further evidence of spontaneous cure in human Chagas disease" de autoria de João Carlos Pinto Dias, Emmanuel Dias, Olindo M. Filho, Danielle Vitelli-Avelar, Dalmo Correia, Eliane Lages e Aluízio Prata, foi publicada o nome do co-autor Olindo M. Filho de forma incorreta, abaixo o nome escrito na forma correta.

\section{Further evidence of spontaneous cure in human Chagas disease}

Uma nova evidência de cura espontânea em doença de Chagas humana

João Carlos Pinto Dias ${ }^{1,3}$, Emmanuel Dias ${ }^{2 \dagger}$, Olindo Assis Martins-Filho ${ }^{3}$, Danielle Vitelli-Avelar ${ }^{3}$, Dalmo Correia ${ }^{4}$, Eliane Lages ${ }^{5}$ and Aluízio Prata ${ }^{4}$ 